\title{
Longitudinal modeling in sports: Young swimmers' performance and biomechanics profile
}

\author{
Jorge E. Morais ${ }^{\mathrm{a}, \mathrm{e}}$, Mário C. Marques ${ }^{\mathrm{b}, \mathrm{e}}$, Daniel A. Marinho ${ }^{\mathrm{b}, \mathrm{e}}$, \\ António J. Silva ${ }^{\mathrm{c}, \mathrm{e}}$, Tiago M. Barbosa ${ }^{\mathrm{d}, \mathrm{e}, *}$ \\ ${ }^{a}$ Department of Sport Sciences, Polytechnic Institute of Bragança, Bragança, Portugal \\ ${ }^{\mathrm{b}}$ Department of Sport Sciences, University of Beira Interior, Covilhã, Portugal \\ ${ }^{\mathrm{C}}$ Department of Sport Sciences, Exercise and Health, University of Trás-os-Montes and Alto Douro, Vila Real, Portugal \\ ${ }^{\mathrm{d}}$ National Institute of Education, Nanyang Technological University, Singapore, Singapore \\ ${ }^{\mathrm{e}}$ Research Centre in Sports, Health and Human Development, Vila Real, Portugal
}

\section{A R T I C L E I N F O}

\section{Article history:}

\section{PsycINFO classification:}

3720

Keywords:

Modeling

Kinematics

Hydrodynamics

Season adaptations

Contribution

\begin{abstract}
A B S T R A C T
New theories about dynamical systems highlight the multifactorial interplay between determinant factors to achieve higher sports performances, including in swimming. Longitudinal research does provide useful information on the sportsmen's changes and how training help him to excel. These questions may be addressed in one single procedure such as latent growth modeling. The aim of the study was to model a latent growth curve of young swimmers' performance and biomechanics over a season. Fourteen boys $(12.33 \pm 0.65$ years-old $)$ and 16 girls $(11.15 \pm 0.55$ years-old $)$ were evaluated. Performance, stroke frequency, speed fluctuation, arm's propelling efficiency, active drag, active drag coefficient and power to overcome drag were collected in four different moments of the season. Latent growth curve modeling was computed to understand the longitudinal variation of performance (endogenous variables) over the season according to the biomechanics (exogenous variables). Latent growth curve modeling showed a high interand intra-subject variability in the performance growth. Gender had a significant effect at the baseline and during the performance growth. In each evaluation moment, different variables had a meaningful effect on performance (M1: $D_{\mathrm{a}}, \beta=-0.62 ; \mathrm{M} 2: D_{\mathrm{a}}$, $\beta=-0.53$; M3: $\eta_{\mathrm{p}}, \beta=0.59$; M4: SF, $\beta=-0.57$; all $P<.001$ ). The models' goodness-of-fit was $1.40 \leqslant \chi^{2} / \mathrm{df} \leqslant 3.74$ (good-reason-
\end{abstract}

\footnotetext{
* Corresponding author at: Physical Education \& Sports Science Academic Group, National Institute of Education, Nanyang Technological University, NIE5-03-31, 1 Nanyang Walk, Singapore 637616, Singapore. Tel.: +65 6219 6213; fax: +65 68969260 .

E-mail address: tiago.barbosa@nie.edu.sg (T.M. Barbosa).
} 
able). Latent modeling is a comprehensive way to gather insight about young swimmers' performance over time. Different variables were the main responsible for the performance improvement. A gender gap, intra- and inter-subject variability was verified.

(c) 2014 Elsevier B.V. All rights reserved.

\section{Introduction}

Talent identification, development, and follow-up are some of the major challenges that sports researchers and practitioners still face nowadays. Swimming performance is characterized by the multi-dimensional interplay of different scientific fields, where a highly complex interaction between several variables exists (Barbosa et al., 2010). Cross-sectional studies reported relationships between young swimmers' performance, Energetics (Toubekis, Vasilaki, Douda, Gourgoulis, \& Tokmakidis, 2011), Biomechanics (Morais et al., 2012) and Motor Control (Silva et al., 2013). Nevertheless, from among all these scientific fields, Biomechanics plays a major role by explaining $50-60 \%$ of the performance of young swimmers (Morais et al., 2012). Probably the partial contribution of each key factor to performance may change across time, for example, over a season. However, until now no longitudinal research has been conducted about it in sports performance. Moreover, longitudinal research should help in gathering insight into: (i) how biomechanical variables interplay and affect performance; (ii) the dynamical changes that happen at these early ages; (iii) the partial contribution of each determinant factor over time.

For a long time sports research was based on the assumption that intra- and inter-subject variability should be minimized. Nowadays, dynamic systems theory and non-linear approaches suggest that variability should not be considered as a random error (Bideault, Herault, \& Seifert, 2013). Evidence has been gathered lately about this topic in adult/elite swimmers (Costa et al., 2013; Komar, Sanders, Chollet, \& Seifert, 2014) even though definitive answers are needed. Besides this, little or almost nothing is known about it in young swimmers. Interestingly young sportsmen, including swimmers, are supposed to be among the ones with a higher variability due to their allegedly low expertise level. It seems that athletes with lower (such as young swimmers) and very high expertise (including elite swimmers) levels are the ones with the highest variability (Seifert et al., 2011).

Until now, classical research designs and data analysis procedures (e.g., analysis of variance and regression models) selected on regular basis in sports performance were not helpful in gathering insight about such highly dynamic and complex relationships. Latent growth curve modeling is a structural equation modeling technique for longitudinal dataset. It is characterized by estimating intra- and inter-subject growth trajectories, enabling researchers to predict future development (Wu, Taylor, \& West, 2009). Structural equation modeling also allows the quantification of how much an exogenous variable contributes to an endogenous variable (Morais et al., 2012). Hence, its potential to explain complex and dynamic changes as reported earlier should be explored. This longitudinal data analysis procedure is reported on regular basis in Social Sciences such as Psychology (Biesanz, West, \& Kwok, 2003; Castellanos-Ryan, Parent, Vitaro, Tremblay, \& Séquin, 2013). In Sport Sciences a couple of papers can be found on physical fitness and health (Maia et al., 2003; Park \& Schutz, 2005) but it was never attempted in sports performance as much as we are aware of.

Therefore, the aim of this study was to model a latent growth curve of young swimmers' performance and biomechanics over a season. It was hypothesized that latent growth curve modeling would explain performance improvement. Different exogenous variables would have a higher contribution on the performance enhancement throughout the season with a significant gender effect. 


\section{Methods}

\subsection{Subjects}

Thirty young swimmers, including 14 boys: $12.33 \pm 0.65-y, 284.85 \pm 67.48$ FINA (Fédération Internationale de Natation) points at the short-course meter (i.e., 25-m length swimming pool) $100-\mathrm{m}$ freestyle; and 16 girls: $11.15 \pm 0.55-\mathrm{y}, 322.56 \pm 45.18$ FINA points at the short-course meter 100 -m freestyle were recruited. All swimmers were in Tanner stages 1-2 by self-report at baseline (Tanner, 1962). The sample included age-group national record holders and champions. The swimmers were part of a national talent ID scheme. At the beginning of the research the swimmers had $3.40 \pm 0.56$ years of training experience. Fig. 1 reports the external training load over the season. Coaches, parents, and/or guardians consented and the athletes assented their participation on this study. All procedures were in accordance to the Helsinki Declaration regarding Human Research. The University of Trás-os-Montes and Alto Douro Ethic Committee also approved the study design (ethic review: UTAD-2011-219).

\subsection{Study design}

The research design (Fig. 2) included repeated measures of kinematic and hydrodynamic variables in four different moments over one season (i.e., longitudinal research). Testing sessions happened immediately before the beginning of the season (baseline-M1), 4 weeks later (first competitionM2), in the middle of the season (24th week-M3) and at the end of the season (38th week-M4). Data collection procedures were carried out in the same conditions at all times (e.g., the same swimming pool, lane, time of day).

\subsection{Theoretical model}

Theoretical model (Fig. 3) was designed to include kinematic, hydrodynamic, and performance, controlling the gender effect. Stroke frequency (SF), intra-cyclic speed fluctuation (dv) and propelling efficiency $\left(\eta_{\mathrm{p}}\right)$ were selected as kinematic outcomes. As for hydrodynamics, active drag $\left(D_{\mathrm{a}}\right)$, coefficient of active drag $\left(C_{\mathrm{Da}}\right)$ and power to overcome drag $\left(P_{\mathrm{d}}\right)$ were selected. Literature reports that

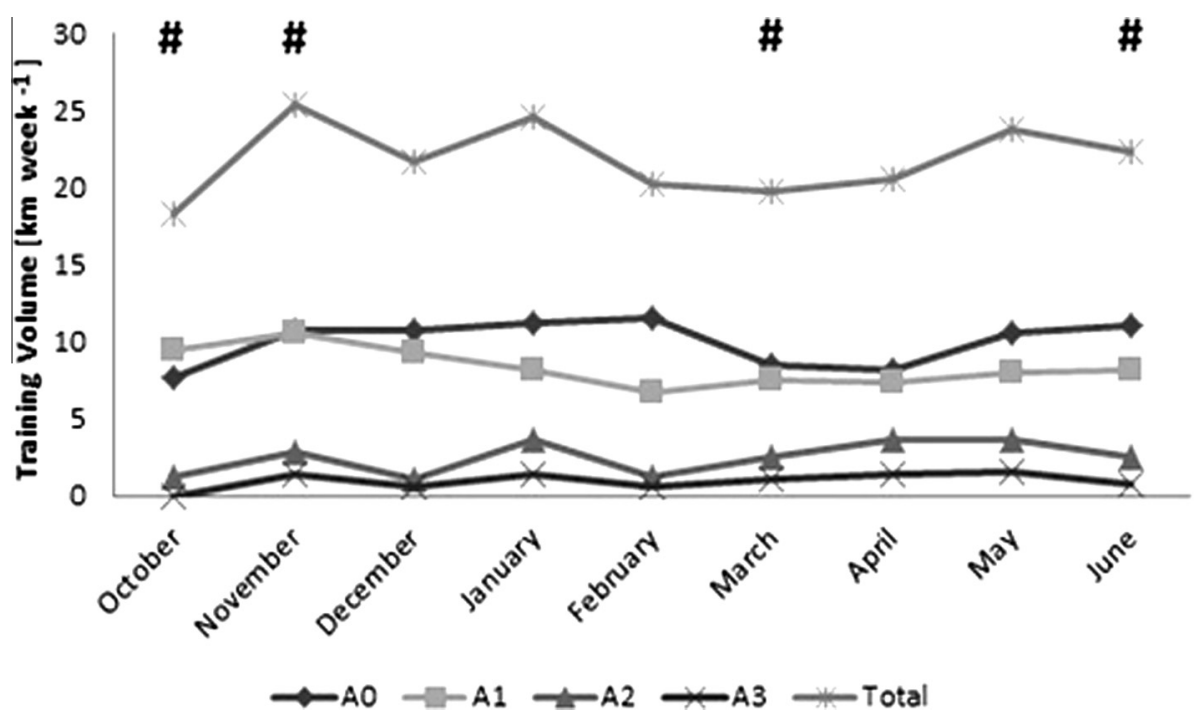

Fig. 1. Training volume per week (in $\mathrm{km}$ ) throughout the season. \# - evaluation moments (Mi); A0 - warm-up and recovery pace; A1 - slow pace; A2 - moderate pace (aerobic capacity); A3 - intense pace (aerobic power). 


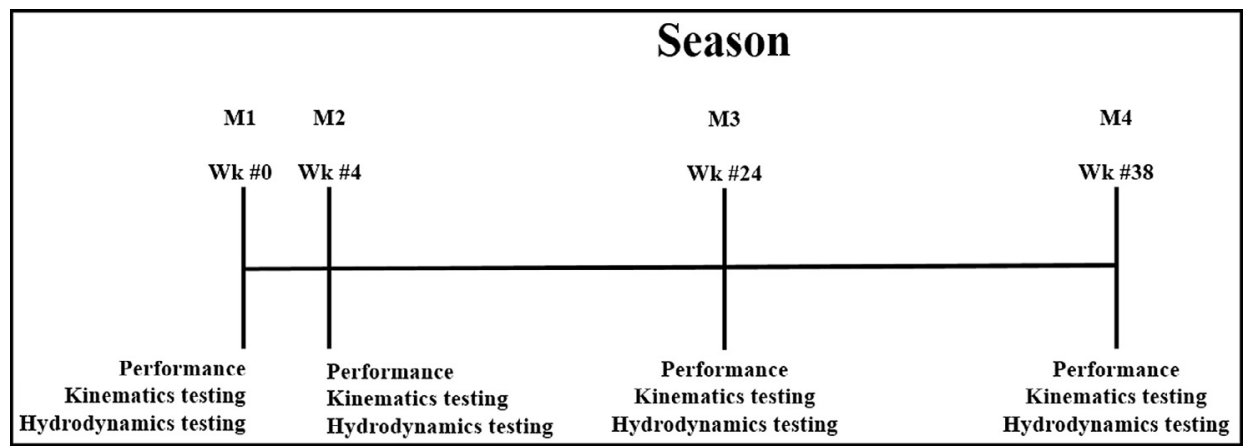

Fig. 2. Study design scheme. M - moment; Wk - week; \# - week's number.

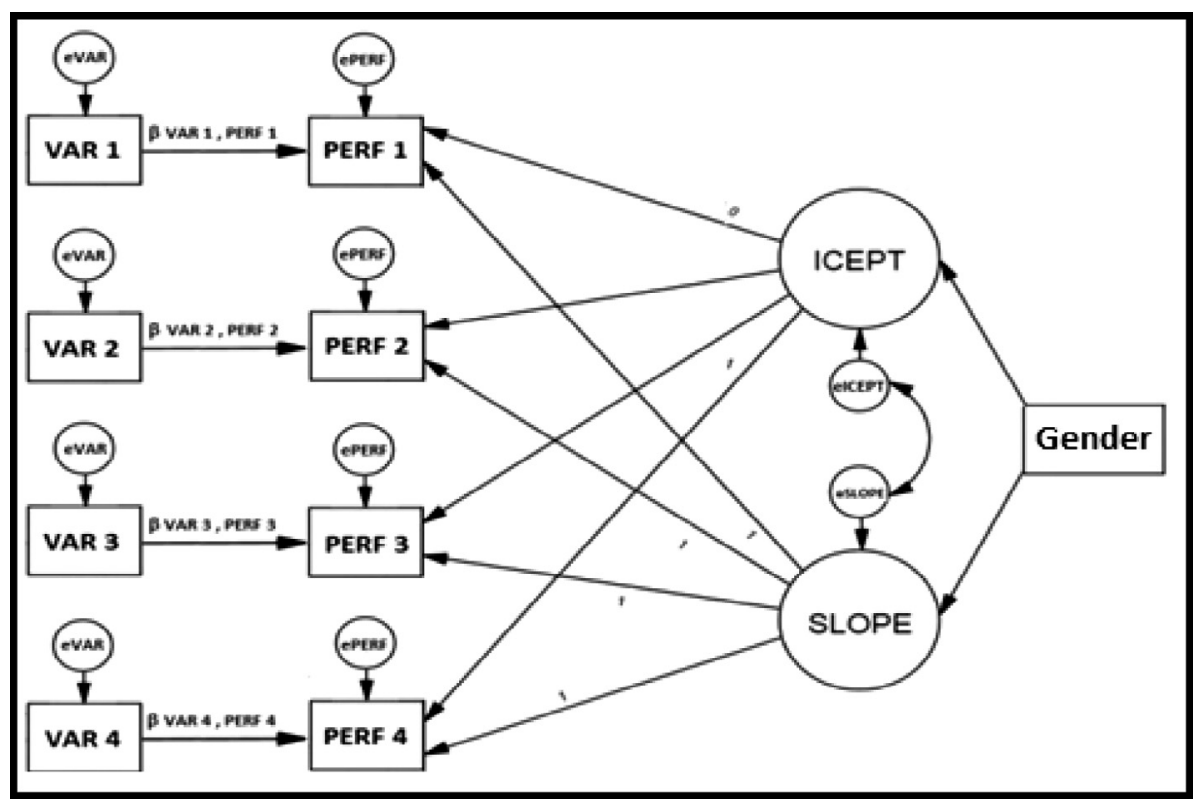

Fig. 3. Theoretical model. VAR (1, 2, 3 and 4) - exogenous variable in M1, M2, M3 and M4, respectively; PERF (1, 2, 3 and 4) performance in M1, M2, M3 and M4, respectively; ICEPT - intercept effect; SLOPE - slope effect; Gender - gender effect; $\beta x_{\mathrm{i}}, y_{\mathrm{i}}$ beta value for regression model between exogenous $\left(x_{\mathrm{i}}\right)$ and endogenous $\left(y_{\mathrm{i}}\right)$ variables; $e_{x \mathrm{i}}$ - disturbance term for a given variable; $x_{\mathrm{i}} \rightarrow y_{\mathrm{i}}$ - variable $y_{\mathrm{i}}$ depends from variable $x_{\mathrm{i}}$.

kinematics and hydrodynamics determine young swimmers' performance (Morais et al., 2012). Stroke frequency, speed fluctuation, and arm's propelling efficiency (i.e., kinematics), active drag, coefficient of active drag, and power to overcome drag (i.e., hydrodynamics) are some of the variables that have a strong relationship with young swimmers' performance and therefore were selected on regular basis in swimming research (Marinho et al., 2010; Morais et al., 2012; Silva et al., 2013).

Swimming performance was chosen as the main outcome (endogenous variable; i.e., dependent variable being predicted), because the primary goal of coaches and swimmers is to enhance the performance. Kinematic and hydrodynamic variables are the exogenous variables (i.e., independent variables that predict the main outcome). The interpretation of this kind of approach is based on: (i) the variables included (inserted inside squares); (ii) the paths (i.e., arrows; an arrow between two variables means that one variable determines the other); (iii) beta values (i.e., these suggest the 
contribution of one variable to the other; when the origin variable increases by one unit the destination variable increases by the amount of the beta value); (iv) residual errors and/or determination coefficient (represents the variable predictive error or the variable predictive value, respectively, in the linked ellipse), and (v) the latent variables (inserted in ellipses) are the no-observed (i.e., the slope analyzes the endogenous variable growth and variability; the intercept analyzes the variability in the baseline).

It was possible to extract the following details from the model: (i) the direct effect (i.e., contribution) of an exogenous variable to the endogenous one (i.e., performance) in each evaluation moment; (ii) the longitudinal growth of the endogenous variable; and (iii) the gender influence at the baseline values (intercept) and also in the endogenous variable growth (i.e., slope).

\subsection{Performance data collection}

The official short course 100-m freestyle race was chosen as performance variable. The time gap between each the race and data collection took no longer than 15-days.

\subsection{Kinematics data collection}

Swimmers were instructed to perform three maximal trials of $25-\mathrm{m}$ at front-crawl with push-off start. Between each trial they had a 30-min rest to ensure full recovery. For further analysis the average value of the three trials was calculated $($ ICC $=0.96)$.

Kinematic data was collected with a mechanical technique (Swim speedo-meter, Swimsportec, Hildesheim, Germany). A 12-bit resolution acquisition card (USB-6008, National Instruments, Austin, Texas, USA) was used to transfer data $(f=50 \mathrm{~Hz})$ to a customized software (LabVIEW ${ }^{\circledR}$ interface, v.2009) (Barbosa et al., 2010). Data were exported to a signal processing software (AcqKnowledge v.3.9.0, Biopac Systems, Santa Barbara, USA) and filtered with a $5 \mathrm{~Hz}$ cut-off low-pass 4th order Butterworth filter. Speed fluctuation was computed as (Barbosa et al., 2010):

$$
\mathrm{dv}=\frac{\sqrt{\sum_{\mathrm{i}}\left(v_{\mathrm{i}}-\bar{v}\right)^{2} F_{\mathrm{i}} / n}}{\sum_{\mathrm{i}} v_{\mathrm{i}} F_{\mathrm{i}} / n}
$$

where $\mathrm{dv}$ is the speed fluctuation, $v$ is the mean velocity, $v_{\mathrm{i}}$ is the instant velocity, $F_{\mathrm{i}}$ is the absolute frequency and $n$ is the number of observations per stroke cycle. Two expert evaluators measured the SF with a stroke counter (base 3 ) and then converted to SI units $\left(\right.$ ICC $=0.98$ ). The $\eta_{p}$ was estimated as (Zamparo, Pendergast, Mollendorf, Termin, \& Minetti, 2005):

$$
\eta_{\mathrm{p}}=\left[\left(\frac{v \cdot 0.9}{2 \pi \cdot \mathrm{SF} \cdot l}\right) \cdot \frac{2}{\pi}\right] \cdot 100
$$

where $\eta_{\mathrm{p}}$ is the arm's propelling efficiency, $v$ is the velocity, SF is the stroke frequency and $l$ is the distance between shoulder and tip of the 3rd finger during the insweep.

\subsection{Hydrodynamics data collection}

The Velocity Perturbation Method was selected to assess the hydrodynamic variables (Kolmogorov \& Duplisheva, 1992). Swimmers performed two extra maximal trials of 25-m at front crawl with pushoff start (one trial with and the other without carrying on the perturbation device). Swimming velocity was assessed between the 11th and 24th $\mathrm{m}$ from the starting wall (Marinho et al., 2010). The time spent to cover this distance was measured with a manual stopwatch (Golfinho Sports MC 815, Aveiro, Portugal) by two expert evaluators (ICC $=0.97$ ). The evaluators followed the swimmer to a have a good line of sight when the swimmer passed the two distance marks. The $D_{\mathrm{a}}$ was estimated as (Kolmogorov \& Duplisheva, 1992):

$$
D_{\mathrm{a}}=\frac{D_{\mathrm{b}} v_{\mathrm{b}} v^{2}}{v^{3}-v_{\mathrm{b}}^{3}}
$$


where $D_{\mathrm{a}}$ is the swimmers' active drag at maximal velocity, $D_{\mathrm{b}}$ is the resistance of the perturbation buoy provided by the, $v_{\mathrm{b}}$ and $v$ are the swimming velocities with and without the perturbation device. The $C_{\mathrm{Da}}$ was calculated as (Kolmogorov \& Duplisheva, 1992):

$$
C_{\mathrm{Da}}=\frac{2 \cdot D_{\mathrm{a}}}{\rho \cdot S \cdot v^{2}}
$$

where $C_{\mathrm{Da}}$ is the active drag coefficient, $\rho$ is the water density (assumed to be $1000 \mathrm{~kg} \mathrm{~m}^{-3}$ ), $v$ is the velocity and $S$ is the swimmers' projected frontal surface area. The $P_{\mathrm{d}}$ was obtained from (Kolmogorov \& Duplisheva, 1992):

$$
P_{\mathrm{d}}=D \cdot v
$$

where $P_{\mathrm{d}}$ is the power to overcome drag, $D$ is the drag and $v$ is the velocity.

\subsection{Statistical procedures}

The normality and homoscedasticity assumptions were analyzed with the Shapiro-Wilk and the Levene tests, respectively. Descriptive statistics included the calculation of the mean, median, minimum, maximum and one standard deviation.

Latent growth curve modeling was used to compute the longitudinal variation of the swimmers' performance over the season. This technique is characterized by estimating intra-individual (represented by the growth parameters; i.e., intercept and slope for growth) in the inter-individual (differences between subjects) growth trajectories (Wu et al., 2009). The intercept and slope are latent variables, which means that they are not directly observed but rather inferred. The intercept determines where the participants' baseline is and how they differ in that specific moment, showing the inter-individual differences between the participants at the baseline, corresponding to M1 in this model). The slope is the average rate of growth, related to the variation throughout a time-frame. It shows the hypothetical differences between the observed moments, and if an inter-individual variability exists or not.

The effect between exogenous ( $\mathrm{SF}, \mathrm{dv}, \eta_{\mathrm{p}}, D_{\mathrm{a}}, C_{\mathrm{Da}}$, and $P_{\mathrm{d}}$ ) and endogenous (performance) variables was also considered. Endogenous variable is the one being predicted and the growth rate analyzed. Exogenous variables are the ones with a direct effect on performance in each evaluation moment. Path-flow analysis model was used to estimate the linear regression standardized coefficients between exogenous and endogenous variables. Standardized regression coefficients $(\beta)$ were selected, and the significance of each one assessed with Student's $t$ test $(P \leqslant .05)$.

The models' goodness-of-fit were measured with the ratio Chi-square/degrees of freedom ( $\left.\chi^{2} / \mathrm{df}\right)$ (Wheaton, 1987). As a rule of thumb if: $5<\chi^{2} / \mathrm{df}$ the model has a poor adjustment; $2<\chi^{2} / \mathrm{df} \leqslant 5$ reasonable adjustment; $1<\chi^{2} / \mathrm{df} \leqslant 2$ good adjustment; $\chi^{2} / \mathrm{df} \sim 1$ very good adjustment.

\section{Results}

Performance improved between the first (M1, $72.05 \pm 5.33 \mathrm{~s}$ ) and last (M4, $66.13 \pm 5.16 \mathrm{~s}$ ) evaluation moments. $D_{\mathrm{a}}$ and $P_{\mathrm{d}}$ showed the highest variations across the season (Table 1 ). Some selected variables $\left(\eta_{\mathrm{p}}, D_{\mathrm{a}}, C_{\mathrm{Da}}\right.$ and $\left.P_{\mathrm{d}}\right)$ increased in a non-linear fashion way.

In M2 and M3, performance achieved 59\% $(P<.001)$ and 99\% $(P<.001)$ of the last evaluation (M4) (Fig. 4). The slope variance was significant for all models, suggesting a heterogeneous growth rate of the performance and hence an inter-subject variability for the pooled sample (i.e., boys plus girls). The $\mathrm{dv}$ model was the one presenting the highest slope $(\beta=6.56 ; P=.003)$. The intercept variance was significant for all models computed, suggesting an inter-subject variability at the baseline for the pooled sample and $\eta_{\mathrm{p}}$ showed the highest intercept $(\beta=28.15 ; P<.001)$. Overall it seems that each participant had its own and unique growth rate suggesting a high inter-subject variability.

Gender had a significant effect on the performance growth with significant paths to intercept and slope for all models (Fig. 4). Both $\eta_{p}$ and $C_{\text {Da }}$ models presented the highest significant paths ( $\beta=0.94$; 
$P<.001)$. Data showed that boys presented better performances than girls. The $P_{\mathrm{d}}$ model had the highest significant path $(\beta=0.86 ; P<.001)$.

All selected variables presented a significant direct effect on performance at least in one evaluation moment (Fig. 4). In M1 the $D_{\text {a }}$ presented the highest direct effect on performance $(\beta=-0.62 ; P<.001$; by each $1 \mathrm{~N}$ increase, performance improved $0.62 \mathrm{~s})$. In $\mathrm{M} 2$ was once again the $D_{\mathrm{a}}(\beta=-0.53 ; P<.001)$, in M3 the $\eta_{\mathrm{p}}(\beta=0.59 ; P<.001)$ and in M4 the $\mathrm{SF}(\beta=-0.57 ; P<.001)$. Hence, swimmers relied on different exogenous variables to enhance performance in different moments of the season.
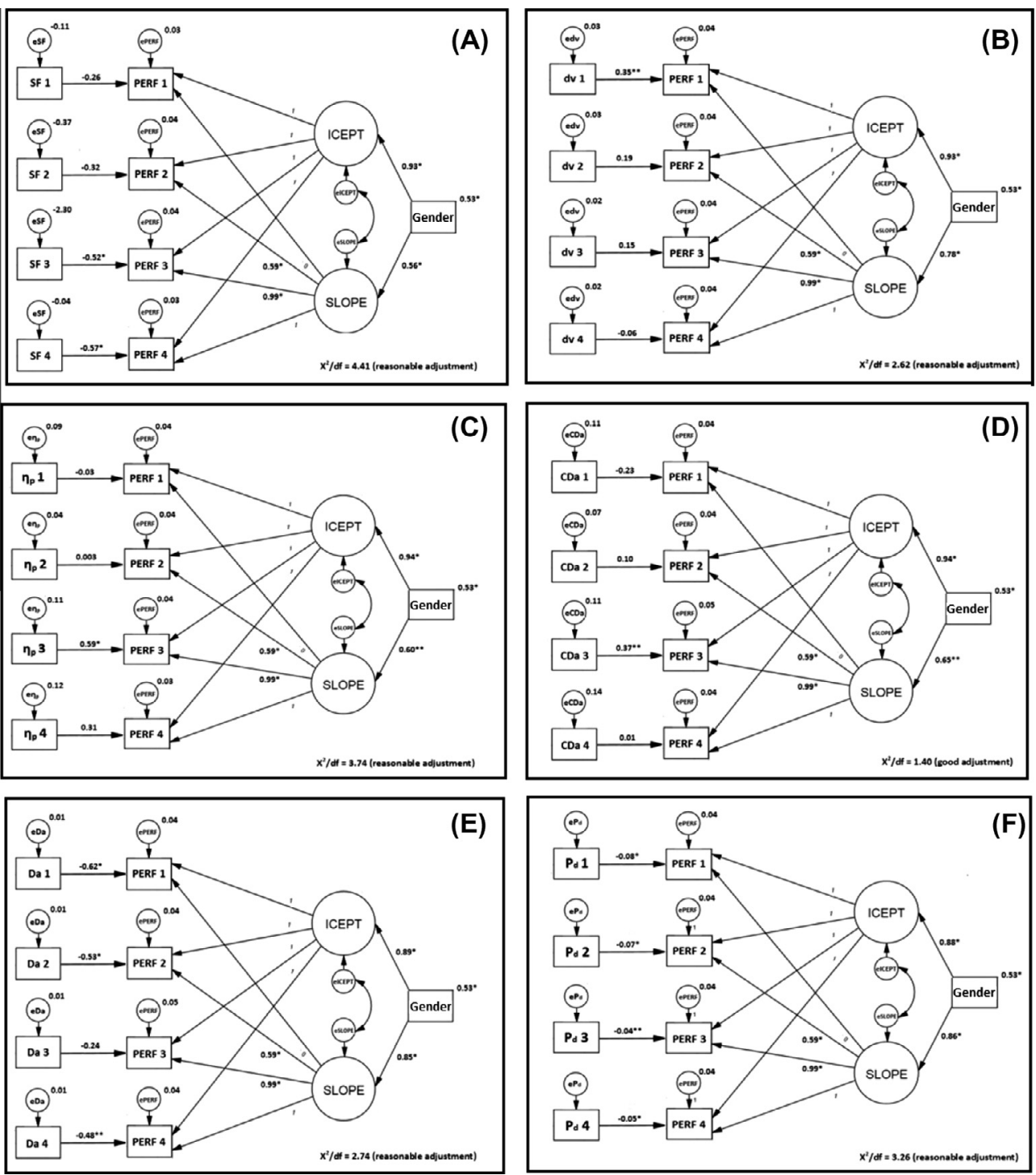

Fig. 4. Growth confirmatory models for performance and effects of the selected variables. (A) SF - stroke frequency; (B) dv speed fluctuation; (C) $\eta_{\mathrm{p}}$ - arm's propelling efficiency; (D) $C_{\mathrm{Da}}$ - active drag coefficient; (E) $D_{\mathrm{a}}$ - active drag; (F) $P_{\mathrm{d}}-$ power to overcome drag; ICEPT - intercept effect; SLOPE - slope effect; Gender - gender effect; $e_{\mathrm{xi}}$ - disturbance term for a given variable; $x_{\mathrm{i}} \rightarrow y_{\mathrm{i}}$ - variable $y_{\mathrm{i}}$ depends from variable $x_{\mathrm{i}}$. 
Table 1

Descriptive statistics for selected kinematic and hydrodynamic variables in each evaluation moment.

\begin{tabular}{|c|c|c|c|c|c|c|c|c|c|c|c|c|c|c|c|c|}
\hline & \multicolumn{4}{|l|}{ PERF [s] } & \multicolumn{4}{|l|}{$\mathrm{SF}[\mathrm{Hz}]$} & \multicolumn{4}{|c|}{ dv [dimensionless] } & \multicolumn{4}{|l|}{$\eta_{\mathrm{p}}[\%]$} \\
\hline & M1 & M2 & M3 & M4 & M1 & M2 & M3 & M4 & M1 & M2 & M3 & M4 & M1 & M2 & M3 & M4 \\
\hline Mean & 72.05 & 68.91 & 66.44 & 66.13 & 0.85 & 0.84 & 0.86 & 0.86 & 0.09 & 0.09 & 0.08 & 0.08 & 30.21 & 30.38 & 33.13 & 31.03 \\
\hline $1 \mathrm{SD}$ & 5.33 & 5.43 & 5.33 & 5.16 & 0.09 & 0.08 & 0.10 & 0.08 & 0.02 & 0.03 & 0.02 & 0.01 & 2.94 & 2.94 & 4.55 & 5.13 \\
\hline Median & 72.02 & 69.32 & 67.43 & 66.66 & 0.83 & 0.83 & 0.83 & 0.83 & 0.09 & 0.09 & 0.08 & 0.08 & 30.47 & 30.78 & 32.12 & 30.73 \\
\hline Minimum & 60.30 & 57.03 & 58.01 & 57.36 & 0.67 & 0.70 & 0.72 & 0.73 & 0.06 & 0.06 & 0.06 & 0.05 & 25.16 & 24.24 & 26.00 & 24.12 \\
\hline \multirow[t]{3}{*}{ Maximum } & 81.00 & 79.12 & 76.85 & 76.06 & 1.05 & 0.99 & 1.07 & 1.03 & 0.16 & 0.17 & 0.14 & 0.10 & 35.80 & 35.06 & 44.99 & 53.06 \\
\hline & \multicolumn{6}{|l|}{$D_{\mathrm{a}}[\mathrm{N}]$} & \multicolumn{5}{|c|}{$C_{\mathrm{Da}}$ [dimensionless] } & \multicolumn{5}{|l|}{$P_{\mathrm{d}}[\mathrm{W}]$} \\
\hline & M1 & \multicolumn{2}{|r|}{ M2 } & M3 & \multicolumn{2}{|l|}{ M4 } & M1 & M2 & M3 & \multicolumn{2}{|l|}{ M4 } & M1 & M2 & \multicolumn{2}{|r|}{ M3 } & M4 \\
\hline Mean & \multicolumn{3}{|r|}{71.01} & 83.25 & \multicolumn{2}{|l|}{75.62} & 0.40 & 0.49 & 0.58 & \multicolumn{2}{|l|}{0.50} & 68.65 & 99.04 & \multicolumn{2}{|r|}{116.93} & 109.62 \\
\hline $1 \mathrm{SD}$ & 27.76 & & 33.70 & 37.30 & \multicolumn{2}{|l|}{36.96} & 0.22 & 0.17 & 0.26 & \multicolumn{2}{|l|}{0.22} & 47.12 & 49.90 & \multicolumn{2}{|r|}{53.83} & 57.08 \\
\hline Median & 34.29 & & 58.36 & 70.85 & \multicolumn{2}{|l|}{77.05} & 0.34 & 0.48 & 0.51 & \multicolumn{2}{|l|}{0.46} & 49.22 & 81.72 & \multicolumn{2}{|r|}{97.36} & 107.25 \\
\hline Minimum & 22.20 & & 26.64 & 22.20 & \multicolumn{2}{|l|}{20.66} & 0.16 & 0.22 & 0.18 & \multicolumn{2}{|l|}{0.18} & 31.34 & 36.55 & & 24.00 \\
\hline Maximum & 139.53 & & 167.33 & 172.93 & \multicolumn{2}{|l|}{189.52} & 1.41 & 0.89 & 1.28 & \multicolumn{2}{|l|}{1.32} & 241.54 & 229.86 & \multicolumn{2}{|r|}{244.27} & 295.41 \\
\hline
\end{tabular}

$\mathrm{Mi}$ - evaluation moment; PERF - performance; SF - stroke frequency; dv - speed fluctuation; $\eta_{\mathrm{p}}$ - arm's propelling efficiency; $D_{\mathrm{a}}$ - active drag; $C_{\mathrm{Da}}$-active drag coefficient; $P_{\mathrm{d}}$ - power to overcome drag. 
The models' goodness-of-fit ranged between $1.40 \leqslant \chi^{2} / \mathrm{df} \leqslant 3.74$ (i.e., good-reasonable). The $C_{\mathrm{Da}}$ model showed highest goodness-of-fit $\left(\chi^{2} / \mathrm{df}=1.40\right.$; good adjustment) and the $\mathrm{SF}$ the lowest one $\left(\chi^{2} / \mathrm{df}=4.41 ;\right.$ reasonable adjustment $)$.

\section{Discussion}

The main aim of this study was to model a latent growth curve of swimming performance and its relationship with biomechanics over time to gather insight about the partial contribution of each factor and the gender effect. In the first two moments, hydrodynamics was the major contributor to performance and in the last two, kinematics. The model was also able to detect a gender gap and a high intra- and inter-subject variability. Therefore, over a season, different determinant factors had a main influence on the performance enhancement for both boys and girls. Besides that, each one of them selected a unique strategy to enhance performance.

Cross-sectional studies showed that young swimmers' performance is highly influenced by kinematics and hydrodynamics (Morais et al., 2012). However, longitudinal follow-up studies that included these variables neglected the inter- and intra-subject changes (Lätt et al., 2009). At least for adult swimming it was pointed out that intra-subject changes are not residual variance and it should not be disregarded in the overall analysis (Connaboy, Coleman, Moir, \& Sanders, 2010; Costa et al., 2013). The same idea was shared earlier by others for motor control (Komar et al., 2014) and kinematics changes (Figueiredo, Seifert, Vilas-Boas, \& Fernandes, 2012; Seifert, Barbosa, \& Kjendlie, 2010; Seifert, Leblanc, Chollet, \& Delignières, 2010). Latent growth curve modeling is able to estimate intra- and inter-subject variability. Variance analysis showed significant differences between swimmers at the baseline and during the performance growth. Residual variances tend to be neglected by other data analysis techniques (e.g., analysis of variance and multi-linear regressions). At least classical techniques are less sensitive to such residual variances. However, those variances are of major interest in latent growth curve modeling (Voelkle, 2007). A main finding of this research was that young swimmers presented a high intra- and inter-subject variability suggesting that each one has a very unique strategy to excel.

Latent growth curve modeling provides the amount of performance that is achieved in intermediate moments. Between M1 and M2 performance reached 59\% of its final value in M4. Between competitive seasons, young swimmers have a break period impairing their energetics and kinematics (Moreira et al., 2014). The improvement between M1 and M2 might be related with the first mesocycle that is characterized by a fairly high volume after the summer break (Fig. 1). Afterwards, performance improved 39\% (between M2 and M3) and 1\% (between M3 and M4). Hence, as the major competition of the season is approaching, improvements are less sharp and meaningful. Similar trend is reported for adult/elite swimmers. Building-up for the major competition, adult swimmers are getting closer from their reserve upper-limits, and it is more challenging for any further improvement (Costa et al., 2013).

A gender gap was also identified at the baseline and during the performance growth. There is a very solid body of knowledge about the gender differences for peri- and post-pubertal athletes (Seifert, Barbosa et al., 2010; Seifert, Leblanc et al., 2010). Literature reports that boys have a higher dv, $D_{\mathrm{a}}$, and SF than girls (Barbosa et al., 2010). Therefore longitudinal structural equation modeling was successful in identifying the well-known gender gap. In this sense, the technique used is also sensitive enough whenever pooled data (both genders) is computed.

Swimming is characterized by the multi-dimensional interplay of different variables that will influence the performance. One might claim that the partial contribution of each exogenous variable to the endogenous one will change over time. That is, the partial contribution of each variable will not be constant over time. However, until now as much as we are aware no paper reported or quantified such phenomenon. Structural equation modeling is very sensitive to such changes and can be used to learn about it. In M1 and M2, $D_{\mathrm{a}}$ was the main performance determinant. Between M1 and M2 periodization included the highest volume of the season (Fig. 1). The goals of those meso-cycles were to build-up energetics (mainly aerobic capacity) and improve technique. It was reported that training based on technical drills and kinesthetic feedbacks improved young swimmers' hydrodynamics and 
performance (Havriluk, 2006). $D_{\mathrm{a}}$ is strongly related to swimming velocity (Eq. (3)). Hence, the increase in speed and therefore in performance lead to a higher $D_{\mathrm{a}}$. $C_{\mathrm{Da}}$ had a minor influence on performance growth. So, it can be speculated that the performance enhancement during this time frame might be more related to energetic build-up and less to technique enhancement.

In $M 3, \eta_{p}$ had the highest direct effect on the performance. Between M2 and M3 periodization was characterized by a decrease in total volume (Fig. 1). These meso-cycles were more focused on technical parameters (enhancing stroke mechanics). This explains why on average the swimmers achieved the highest $\eta_{\mathrm{p}}$ in M3. Since long there has been a discussion whether young swimmers training should rely more on energetics or efficiency. Cross-sectional confirmatory models suggested that $50-60 \%$ of performance in these age-groups is related to biomechanics and technique enhancement (Morais et al., 2012). In M4, SF was the variable presenting the highest direct effect on performance. Between M3 and M4 periodization included an increase in the aerobic power and aerobic capacity sets (Fig. 1). This was coupled with a slight increase of the dry-land training sessions that included strength power routines. For adolescent sprint swimmers, an association was found between high muscular strength parameters and an increase in SF (Girould, Maurin, Dugué, Chatard, \& Millet, 2007). At least in adult swimmers aerobic power paces are related to customize SF-stroke length relationships (McLean, Palmer, Ice, Truijens, \& Smith, 2010; Wakayoshi, D’Acquisito, Cappert, \& Troup, 1995). Therefore, to swim at aerobic power sets a fairly constant and high stroke length with a high SF is needed. To be able to optimize this SF-stroke length relationship dry-land power training is a must.

It was attempted in one of the earliest models to include anthropometrics variables to control the potential confounding factor of the maturation and growth. However, after running the model, we failed to obtain significant results and a reasonable adjustment. Because we track down and followup subjects in Tanner 1-2, one might consider that most of them are yet pre-pubescent and therefore one single year is not enough to verify significant changes in biological maturation. With this we are not suggesting that they are not in a process of biological development but only that because they did not reach any spur, it is more challenging to have anthropometrics as a determinant factor. However, later one, that is, swimmers in the following Tanner stages this is more obvious (Falk, Bronshtein, Zigel, Constantini, \& Eliakim, 2004; Jurimae et al., 2007). Overall, in M1 and M2 hydrodynamics (i.e., $D_{\mathrm{a}}$ ) was the major contributor to performance while in M3 and M4 was the kinematics ( $\eta_{\mathrm{p}}$ and SF, respectively). Therefore, the main determinant at a given moment is related to the periodization model designed. It is possible to design models that are more based on energetics (M1 and M2) or technique (M3 and M4). A model that relies more on energetics allows a very quick and sharp improvement, but on the other hand the efficiency is compromised and increases the odds of an early burn-out. A model that is based on the technique is more time-consuming and performance enhancement might take some time to happen. However, a proper technique will be needed for further improvement reaching adulthood, when most of the periodization is energetically oriented (e.g., Schnitzler, Seifert, Chollet, \& Toussaint, 2014). Besides, it is at these early ages that the motor learning mechanisms of any skill is more effective. Considering the pros and cons of each approach, an agegroup coach should consider to compromise both (energetics build-up and technique enhancement) but putting more focus on the technique and efficiency if the athlete's career is to be seen in the long-run. Hence, it seems that many of the changes in performance can be attributed to the type of training that swimmers were undergoing at the time of each data collection. This could be useful for coaches as it shows that technical parameters are the most determinant ones in the young swimmers' performance improvement. They can apply these technical drills according to their macrocycles, avoiding the athletes to burn out with high amounts of training workloads, especially close to the main events.

\section{Conclusion}

Latent modeling is a comprehensive way to gather insight about young swimmers' performance over time. This was showcased with young swimmers engaged in a national talent ID scheme. Different variables were responsible for the performance improvement over the season. A significant intraand inter-subject variability was verified. These findings suggest that a very unique and customized 
strategy is used by each swimmer to excel. Overall it seems that young swimmers coaches' should put the focus on the hydrodynamic profile and also on the stroke mechanics (i.e., technical ability) to enhance the performance, notably sprinters. Moreover the performance main determinants are also related to the training periodization.

\section{Conflict of interest}

The authors have no professional relationships to disclose with companies or manufacturers who will benefit from the results of the present study.

\section{Acknowledgments}

Jorge E. Morais gratefully acknowledges the Ph.D. scholarship granted by the Portuguese Science and Technology Foundation (FCT) (SFRH/BD/76287/2011). The authors wish to thanks Pedro Forte and Marc Moreira for their useful help during data collection.

\section{References}

Barbosa, T. M., Bragada, J. A., Reis, V. M., Marinho, D. A., Carvalho, C., \& Silva, A. J. (2010). Energetics and biomechanics as determining factors of swimming performance: Updating the state of the art. Journal of Science and Medicine in Sport, 13, 262-269.

Bideault, G., Herault, R., \& Seifert, L. (2013). Data modelling reveals inter-individual variability of front crawl swimming. Journal of Science and Medicine in Sport, 16, 281-285.

Biesanz, J. C., West, S. G., \& Kwok, O. M. (2003). Personality over time: Methodological approaches to the study of short-term and long-term development and change. Journal of Personality, 71, 905-941.

Castellanos-Ryan, N., Parent, S., Vitaro, F., Tremblay, R. E., \& Séquin, J. R. (2013). Pubertal development, personality, and substance use: A 10 year longitudinal study from childhood to adolescence. Journal of Abnormal Psychology, 122, $782-796$.

Connaboy, C., Coleman, S., Moir, G., \& Sanders, R. (2010). Measures of reliability in the kinematics of maximal undulatory underwater swimming. Medicine and Science in Sports and Exercise, 42, 762-770.

Costa, M. J., Bragada, J. A., Mejias, J. E., Louro, H., Marinho, D. A., Silva, A. J., et al (2013). Effects of swim training on energetics and performance. International Journal of Sports Medicine, 34, 507-513.

Falk, B., Bronshtein, Z., Zigel, L., Constantini, N., \& Eliakim, A. (2004). Higher tibial quantitative ultrasound in young female swimmers. British Journal of Sports Medicine, 38, 461-465.

Figueiredo, P., Seifert, L., Vilas-Boas, J. P., \& Fernandes, R. J. (2012). Individual profiles of spatio-temporal coordination in high intensity swimming. Human Movement Science, 31, 1200-1212.

Girould, S., Maurin, D., Dugué, B., Chatard, J. C., \& Millet, G. (2007). Effects of dry-land vs. resisted- and assisted-sprint exercises on swimming sprint performances. Journal of Strength and Conditioning Research, 21, 599-605.

Havriluk, R. (2006). Magnitude of the effect of an instructional intervention on swimming technique and performance. In J. P. Vilas-Boas, F. Alves, \& A. Marques (Eds.), X International symposium of biomechanics and medicine in swimming (pp. 218-220). Porto: Portuguese Journal of Sport Sciences.

Jurimae, J., Haljaste, K., Cicchella, A., Latt, E., Purge, P., Leppik, A., et al (2007). Analysis of swimming performance from physical, physiological, and biomechanical parameters in young swimmers. Pediatric Exercise Science, 19, 70-81.

Kolmogorov, S., \& Duplisheva, O. (1992). Active drag, useful mechanical power output and hydrodynamic force in different swimming strokes at maximal velocity. Journal of Biomechanics, 25, 311-318.

Komar, J., Sanders, R. H., Chollet, D., \& Seifert, L. (2014). Do qualitative changes in inter-limb coordination lead to effectiveness of aquatic locomotion rather than efficiency? Journal of Applied Biomechanics, 30, 189-196.

Lätt, E., Jürimäe, J., Haljaste, K., Cicchella, A., Purge, P., \& Jürimäe, T. (2009). Longitudinal development of physical and performance parameters during biological maturation of young male swimmers. Perceptual Motor Skills, 108, 297-307.

Maia, J. A., Beunen, G., Lefevre, J., Claessens, A. L., Renson, R., \& Vanreusel, B. (2003). Modeling stability and change in strength development: A study in adolescent boys. American Journal of Human Biology, 15, 579-591.

Marinho, D. A., Barbosa, T. M., Costa, M. J., Figueiredo, C., Reis, V. M., Silva, A. J., et al (2010). Can 8 weeks of training affect active drag in young swimmers? Journal of Sports Science and Medicine, 9, 71-78.

McLean, S. P., Palmer, D., Ice, G., Truijens, M., \& Smith, J. C. (2010). Oxygen uptake response to stroke rate manipulation in freestyle swimming. Medicine and Science in Sports and Exercise, 42, 1909-1913.

Morais, J. M., Jesus, S., Lopes, V., Garrido, N., Silva, A. J., Marinho, D. A., et al (2012). Linking selected kinematic, anthropometric and hydrodynamic variables to young swimmer performance. Pediatric Exercise Science, 24, 649-664.

Moreira, M., Morais, J. E., Marinho, D. A., Silva, A. J., Barbosa, T. M., \& Costa, M. J. (2014). Growth influences biomechanical profile of talented swimmers during the summer break. Sports Biomechanics, 13, 62-74.

Park, I., \& Schutz, R. W. (2005). An introduction to latent growth models: Analysis of repeated measures physical performance data. Research Quarterly for Exercise and Sport, 76, 176-192.

Schnitzler, C., Seifert, L., Chollet, D., \& Toussaint, H. (2014). Effect of aerobic training on inter-arm coordination in highly trained swimmers. Human Movement Science, 33, 43-54.

Seifert, L., Barbosa, T. M., \& Kjendlie, P. L. (2010). Biophysical approach to swimming: Gender effect. In S. A. Davies (Ed.), Gender gap: causes, experiences and effects (pp. 59-80). New York: Nova Science Publishers. 
Seifert, L., Leblanc, H., Chollet, D., \& Delignières, D. (2010). Inter-limb coordination in swimming: Effect of speed and skill level. Human Movement Science, 29, 103-113.

Seifert, L., Leblanc, H., Herault, R., Komar, J., Button, C., \& Chollet, D. (2011). Inter-individual variability in the upper-lower limb breaststroke coordination. Human Movement Science, 30, 550-565.

Silva, A. F., Figueiredo, P., Seifert, L., Soares, S., Vilas-Boas, J. P., \& Fernandes, R. J. (2013). Backstroke technical characterization of 11-13 year old swimmers. Journal of Sports Science and Medicine, 12, 623-629.

Tanner, J. M. (1962). Growth at adolescence (2nd ed.). Oxford: Blackwell Scientific.

Toubekis, A. G., Vasilaki, A., Douda, H., Gourgoulis, V., \& Tokmakidis, S. (2011). Physiological responses during interval training at relative to critical velocity intensity in young swimmers. Journal of Science and Medicine in Sport, 14, 363-368.

Voelkle, M. C. (2007). Latent growth curve modeling as an integrative approach to the analysis of change. Psychology Science, 49 , 375-414.

Wakayoshi, K., D’Acquisito, J., Cappert, J. M., \& Troup, J. P. (1995). Relationship between oxygen uptake, stroke rate and swimming velocity in competitive swimming. International Journal of Sports Medicine, 16, 19-23.

Wheaton, B. (1987). Assessment of fit in overidentified models with latent variables. Sociological Methods Research, 16, 118-154.

Wu, W., Taylor, A. B., \& West, S. G. (2009). Evaluating model fit for growth curve models: Integration of fit indices from SEM and MLM frameworks. Psychological Methods, 14, 183-201.

Zamparo, P., Pendergast, D. R., Mollendorf, J., Termin, A., \& Minetti, A. E. (2005). An energy balance of front crawl. European Journal of Applied Physiology, 94, 134-144. 